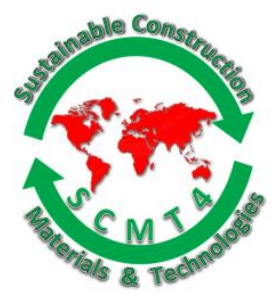

SCMT4

Las Vegas, USA, August 7-11, 2016

\title{
Study on Physical Property of the Modified Fly Ash Controlled Modifying Levels and Fresh Property of the Modified Fly Ash Slurry Mortar
}

\author{
Shoma Takegawa ${ }^{1 a}$, Koji Takasu ${ }^{1 b}$, Hidehiro Koyamada ${ }^{1 c}$, and Hiroki Suyama ${ }^{1 d}$ \\ ${ }^{1}$ The University of Kitakyusyu - 1-1 Hibikino Wakamatsu-ku Kitakyushu, Fukuoka, 808-0135, Japan, \\ ${ }^{1 a}$ Email: <w5mbb016@eng.kitakyu-u.ac.jp >, ${ }^{1 b}$ Email: <takasu@kitakyu-u.ac.jp>,

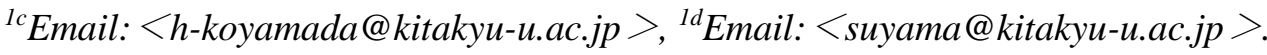

\begin{abstract}
In study of the past, we developed the device removing unburnt carbon from the fly ash by flotation method and examined the performance of the device by experiments. It was succeeded remove of unburnt carbon in the fly ash and to clean the surface of the fly ash by used the developed device removing unburnt carbon in the fly ash by flotation method. As a result, we improved low quality fly ash to equivalent JIS A 6201 class II by used flotation method and, examined the optimum modifying condition of the low quality fly ash. We revealed optimum modifying time and optimum agent addition rate per mass of fly ash. We made concrete with modified fly ash slurry.
\end{abstract}

In this study, We confirmed a relationship between the quality of modified fly ash slurry controlled the modifying levels and mortar properties. As a result of experiment, We revealed the fly ash which was not suitable for modifying it. On the other hand, it was clear that We could pre-evaluate the flow level of mortar using the modified fly ash slurry in considering both loss on ignition and BET specific surface area. Thepercent flow adapted JIS class and it was more than $99 \%$ by using fly ash slurry which loss on ignition was less than approx. 4.3\%, and BET specific surface area was less than approx. $4.5 \mathrm{~m} 2 / \mathrm{g}$. Because we confirmed the fly ash which adsorbed water, method to using the fly ash slurry which adsorbed water was effective for reduction of the quantity of agent addition.

\section{INTRODUCTION}

To promote the use of low-quality fly ash as a concrete admixture, we are developing and studying flotation techniques for quality improvement and stabilization of fly ash. The fly ash modified in the flotation equipment is discharged as a slurry, which is mixed with water in the flotation process. Because there are few practical examples of using fly ash slurry as a mixture material, it is necessary to carefully research and examine the use of processing methods for modified fly ash slurry.Accordingly, in this study we investigated the quality change of fly ash in the modification and slurrying processes and the characteristics of mortar using the fly ash slurry, and we examined the usefulness of controlled coal ash slurry (CCAS) and the slurrying process.

\section{METHOD OF TESTING}

Table 1 shows the physical properties of fly ash we used. Increase and decrease from original fly ash of the physical properties show by this mark." $\Delta \boldsymbol{\nabla}$ " This experiment used six types of fly ash (a-f) 
discharged from various sources, among which fly ash type f is a JIS class II certified product. In modifying the original ash, the modifying level was controlled by processing parameters (preprocessing time, amount of chemical agents, floating time) so that multiple types of modified ash were made from one type of original ash. The modified fly ash was classified into the three types (L, M, and S) according to ignition loss. Fifteen types of modified fly ash were prepared: three types (L, M, and S) from each of the four fly ash types a-d "each of the four" was added to indicate a-L, a-M, a-S, b-L, b-M, etc., to clearly indicate $3 \times 4=12$ types, two types ( $\mathrm{L}$ and $\mathrm{M}$ ) from fly ash type e, and one type (S) from fly ash type $\mathrm{f}$. In addition, in this experiment, 15 types of CCAS were fabricated over a period of approximately two weeks, resulting in various durations of slurry storage. Therefore, to prevent quality variations due to different storage periods, CCAS was dried in a drying oven at 105 degrees just after fabrication. The fly ash was then stored as a dry powder and slurried again when pouring concrete. The percent flow and strength activity index were measured based on JIS A 6201. All 21 types of fly ash shown in Table 1 were used in the examination of the percent flow, while only the 15 types of modified fly ash were used in the examination of the strength activity index and other material properties. In addition, when adding fly ash slurry as a mixture material, the fly ash concentration was set at $60 \%$ to reduce the influence of fly ash precipitation. Then, the fly ash was slurried again for $10 \mathrm{~min}$ using a stirrer at a rotational speed of $400 \mathrm{rpm}$. Since CCAS contains water, mixing was performed to compensate for the water loss and maintain the unit water amount needed for the blend.

Table 1. Properties of fly ash

\begin{tabular}{|c|c|c|c|c|c|c|c|c|}
\hline Fly ash & Mark & $\begin{array}{c}\text { Loss on } \\
\text { Ignition[\%] }\end{array}$ & $\begin{array}{l}\text { Density } \\
{\left[\mathrm{g} / \mathrm{cm}^{3}\right]}\end{array}$ & $\mathrm{SiO}_{2}[\%]$ & $\begin{array}{c}\text { BET specific } \\
\text { surface } \\
\text { area }\left[\mathrm{m}^{2} / \mathrm{g}\right]\end{array}$ & $\begin{array}{c}\text { Blaine } \\
\text { specific } \\
\text { surface area } \\
{\left[\mathrm{cm}^{2} / \mathrm{g}\right]}\end{array}$ & $\begin{array}{c}\text { specific } \\
\text { surface area } \\
{\left[\mathrm{cm}^{2} / \mathrm{cm}^{3}\right]}\end{array}$ & $\begin{array}{c}\text { average } \\
\text { particle } \\
\text { diameter } \\
{[\mu \mathrm{m}]}\end{array}$ \\
\hline \multirow{4}{*}{ FA-a } & $\mathrm{a}$ & 12.82 & 2.22 & 62.38 & 5.340 & 4740 & 17286 & 16.07 \\
\hline & $a-L$ & 6.28 & 2.23 & $61.99 \nabla$ & $6.745 \triangle$ & $4130 \nabla$ & $18547 \triangle$ & $23.13 \triangle$ \\
\hline & $\mathrm{a}-\mathrm{M}$ & 4.23 & 2.24 & $62.42 \triangle$ & $4.458 \boldsymbol{\nabla}$ & $4310 \boldsymbol{\nabla}$ & $21806 \triangle$ & $19.00 \triangle$ \\
\hline & $\mathrm{a}-\mathrm{S}$ & 2.32 & 2.18 & $65.34 \triangle$ & $3.179 \nabla$ & $4690 \nabla$ & $23813 \triangle$ & $17.77 \triangle$ \\
\hline \multirow{4}{*}{ FA-b } & $\mathrm{b}$ & 9.63 & 2.18 & 53.45 & 4.124 & 3880 & 22994 & 18.11 \\
\hline & $\mathrm{b}-\mathrm{L}$ & 6.08 & 2.16 & $53.64 \triangle$ & $2.599 \nabla$ & $4080 \triangle$ & $22351 \nabla$ & $25.57 \triangle$ \\
\hline & b-M & 4.18 & 2.17 & $54.22 \triangle$ & $2.856 \nabla$ & $4360 \triangle$ & $25732 \triangle$ & $21.88 \triangle$ \\
\hline & $\mathrm{b}-\mathrm{S}$ & 2.06 & 2.21 & $55.71 \triangle$ & $2.531 \nabla$ & $4410 \triangle$ & $27053 \triangle$ & $20.65 \triangle$ \\
\hline \multirow{4}{*}{ FA-c } & $\mathrm{c}$ & 5.66 & 2.11 & 68.28 & 1.990 & 4410 & 12357 & 21.39 \\
\hline & $\mathrm{c}-\mathrm{L}$ & 4.33 & 2.17 & $69.41 \triangle$ & $3.501 \triangle$ & $4250 \nabla$ & $16935 \triangle$ & $21.41 \triangle$ \\
\hline & $\mathrm{c}-\mathrm{M}$ & 0.73 & 2.17 & $68.56 \triangle$ & $2.206 \triangle$ & $4330 \nabla$ & $16181 \triangle$ & $17.31 \nabla$ \\
\hline & $\mathrm{c}-\mathrm{S}$ & 0.38 & 2.20 & $72.30 \triangle$ & $2.002 \triangle$ & $4280 \boldsymbol{\nabla}$ & $16039 \triangle$ & $17.31 \nabla$ \\
\hline \multirow{4}{*}{ FA-d } & $\mathrm{d}$ & 5.22 & 2.25 & 65.93 & 4.715 & 3960 & 9277 & 15.55 \\
\hline & $\mathrm{d}-\mathrm{L}$ & 2.74 & 2.21 & $66.60 \triangle$ & $3.144 \nabla$ & $3950 \nabla$ & $8633 \nabla$ & $22.52 \triangle$ \\
\hline & $\mathrm{d}-\mathrm{M}$ & 1.61 & 2.19 & $65.15 \nabla$ & $1.819 \nabla$ & $4120 \triangle$ & $8904 \nabla$ & $20.61 \triangle$ \\
\hline & $\mathrm{d}-\mathrm{S}$ & 0.50 & 2.22 & $68.69 \triangle$ & $1.929 \nabla$ & $4360 \triangle$ & $8626 \nabla$ & $20.47 \triangle$ \\
\hline \multirow{3}{*}{ FA-e } & $\mathrm{e}$ & 5.47 & 2.24 & 64.28 & 3.214 & 4720 & 21275 & 14.74 \\
\hline & e-L & 4.60 & 2.21 & $63.26 \nabla$ & $9.661 \triangle$ & $3990 \nabla$ & $17312 \nabla$ & $17.45 \triangle$ \\
\hline & e-M & 2.27 & 2.25 & $62.25 \nabla$ & $14.015 \triangle$ & $4090 \nabla$ & $18248 \nabla$ & $15.91 \triangle$ \\
\hline \multirow[t]{2}{*}{ FA-f } & $\begin{array}{c}\mathrm{f} \\
(\mathrm{JIS} \text { class II })\end{array}$ & 1.67 & 2.43 & 58.34 & 1.037 & 4270 & 12286 & 10.07 \\
\hline & $\mathrm{f}-\mathrm{S}$ & 0.88 & 2.39 & $59.38 \triangle$ & $2.182 \triangle$ & $4340 \triangle$ & $13188 \triangle$ & $9.42 \boldsymbol{\nabla}$ \\
\hline
\end{tabular}

\section{EXPERIMENTAL RESULTS AND DISCUSSION}

Relationship between CCAS's Properties and Percent Flow. Figure 1 shows percent flow. Except for ash types e-L and e-M, it was found that the percent flow could be improved through the modification of fly ash. Data on modifying level show that the percent flow increased with modifying 
level in 12 of 13 blends, except d-L. Among the modified fly ash types with a percent flow of less than $95 \%$, the ignition loss was found to be greater than $6.0 \%$ in types a, a-L, b, and b-L. However, the percent flows for modified fly ash types e- $\mathrm{L}$ and e-M were small despite their relatively small ignition loss $(4.60 \%$ and $2.27 \%$, respectively). It is reasonable to assume that the percent flow was not only influenced by the ignition loss but also strongly influenced by other properties of fly ash.

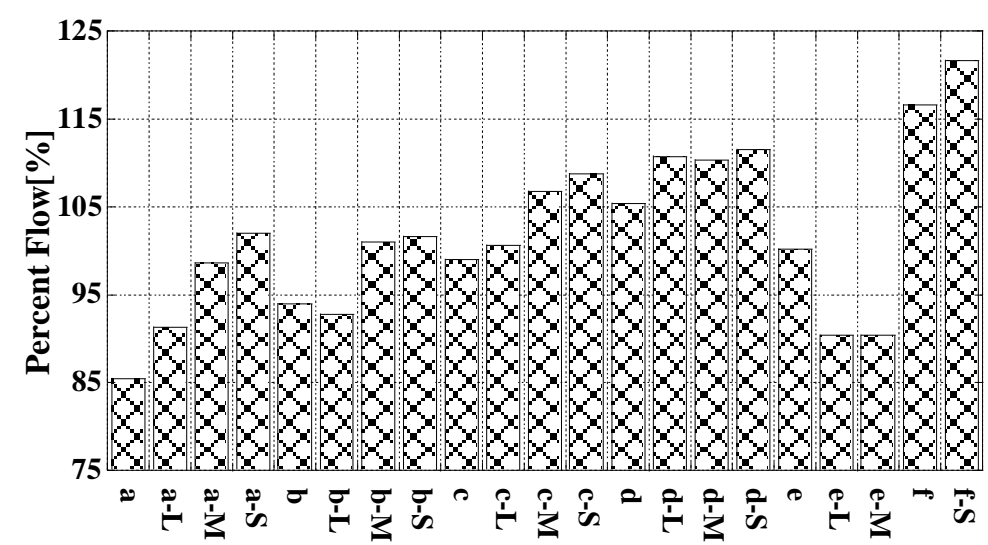

Figure 1. percent flow

Figure 2 shows the relationship between percent flow and loss on ignition, percent flow and BET specific surface area. A negative correlation was found between these parameters[[percent flow and ignition loss]], and the coefficients of determination were 0.54 and 0.40 [[ for types e-L and e-M]], respectively. Moreover, it has been is reported that larger BET specific surface areas adversely affect the fluidity of fly ash if the value is greater than $3.60 \mathrm{~m} 2 / \mathrm{g}$. This experiment showed that fluidity was adversely affected in types a, a-L, e-L, and e-M, which had the BET specific surface areas much greater than $3.60 \mathrm{~m} 2 / \mathrm{g}$, even though their percent flow were as low as $85-91 \%$. These observations suggest that the percent flow can be made to exceed $99 \%$ by using a modified fly ash slurry with ignition loss of about $4.3 \%$ and BET specific surface area of less than $4.5 \mathrm{~m} 2 / \mathrm{g}$. Thus, the possibility of meeting the JIS class II standard is high. It is clear that the flow value of CCAS can be appropriately evaluated by considering both ignition loss and BET specific surface area.
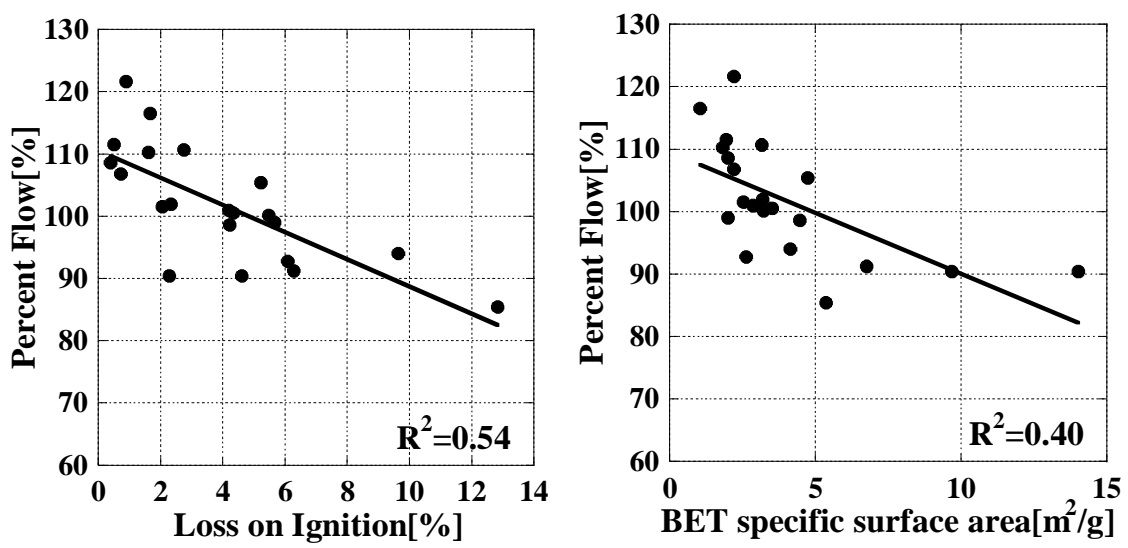

Figure 2. Relationship between percent flow and loss on ignition

\section{BET Specific Surface Area}

As seen in fly ash type e, the percent flow of which remarkably decreased with modification, the adverse effects produced by slurrying fly ash should also be considered. Horiuchi reported that by immersing fly ash in water and leaving it for 1-14 days, compressive strength decreased and reactants formed on 
the particle surface. Shimazaki slurried fly ash and kept stirring it for 1-56 days and found that reactants formed on the particle surface, though the flow value ratio increased only slightly and strength was unaffected. The different effects observed depending on whether fly ash is left unattended or stirred indicate the necessity of finding ways to select fly ash types suitable for flotation.

In this experiment, as shown by the data in Table 1, the BET specific surface area increased compared with that of the original fly ash when we slurried modified fly ash types a-L, c-L, e-L, and f-S (with the exception of types $b$ and $d$ ), which were characterized by low modifying level. Notably, the BET specific surface area of fly ash type e-M was large even with its especially high modifying level. This particular property is different from that of the other fly ash types tested. We found that a BET specific surface area of greater than $3.60 \mathrm{~m} 2 / \mathrm{g}$ in the original fly ash (except fly ash type e) could be reduced to below $3.2 \mathrm{~m} 2 / \mathrm{g}$ by removing unburned carbon through modification of more than a medium modifying level. Therefore, using the type of modification that reduces voids created by unburned carbon, we can reduce the water adsorption in carbon and improve fluidity.

Figure 31 shows SEM images of a, a-L, e and e-L. Unburned carbon adhered to particles of original fly ash types a and e. Images of modified fly ash types a-L and e-L show that the particle surface structure of ash type e-L was specifically different. Although the surface structure of type e-M was found to be similar to that of type e-L, such a structure was not observed in original fly ash type e. Therefore, it is thought that by immersing fly ash type e in water for modification, a hydrate was produced that caused a change of state. It is reasonable to assume that this mechanism causes the BET specific surface area of modified ash types e-L and e-M to increase compared with that of the original fly ash. In summary, we found that immersing fly ash in water greatly affects the BET specific surface area and that the increase in the BET specific surface area by immersion points to the possibility of hydrate formation, which adversely affects the flow value.

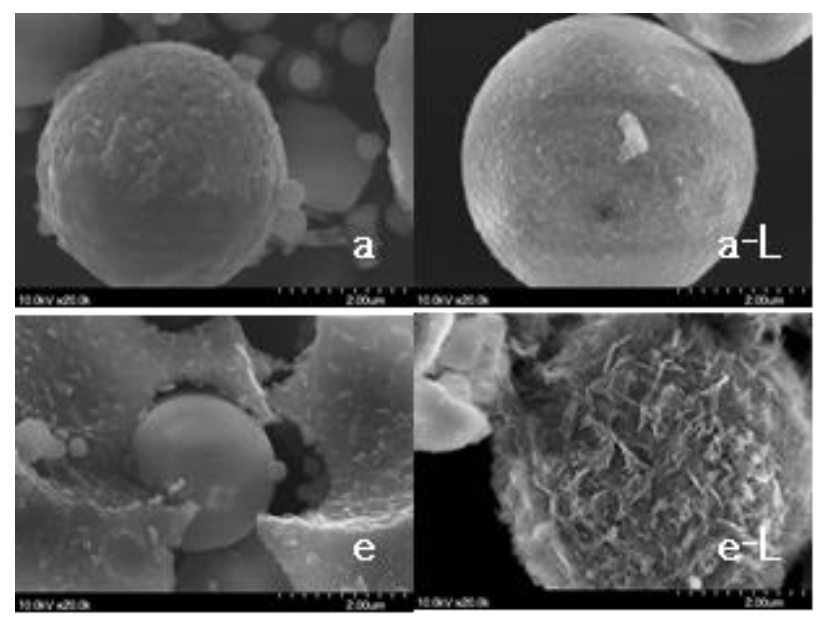

Figure 3. SEM images

Strength Activity Index. Figure 4 shows the strength activity index on 7, 28 and 91 days of material age. The experimental results show that strength increases with material age in the b-L blend except from 7 to 28 days. For modified fly ash types b-L, c-L, and e-M, strength at a material age of 28 days remained lower than $80 \%$ of the standard strength activity index for JIS classes II and III. In addition, at the same material age, no fly ash type exceeded the strength activity index of $90 \%$, which is the standard for JIS class I. At a material age of 91 days, an increase of long-term strength was observed, and all fly ash types exceeding the strength activity index of $90 \%$, which is the standard strength activity index for JIS classes II and III for that age. In addition, eight fly ash types achieved an strength activity index of $100 \%$, which is the standard for JIS class I. These results show that strength remarkably increased between 28 and 91 days in this experiment. 
In addition, no clear relationship was found between the strength activity index and a change in material properties. Therefore, we considered that the quality change caused by fly ash modifying level affected the flow value but not the strength activity index in this experiment.

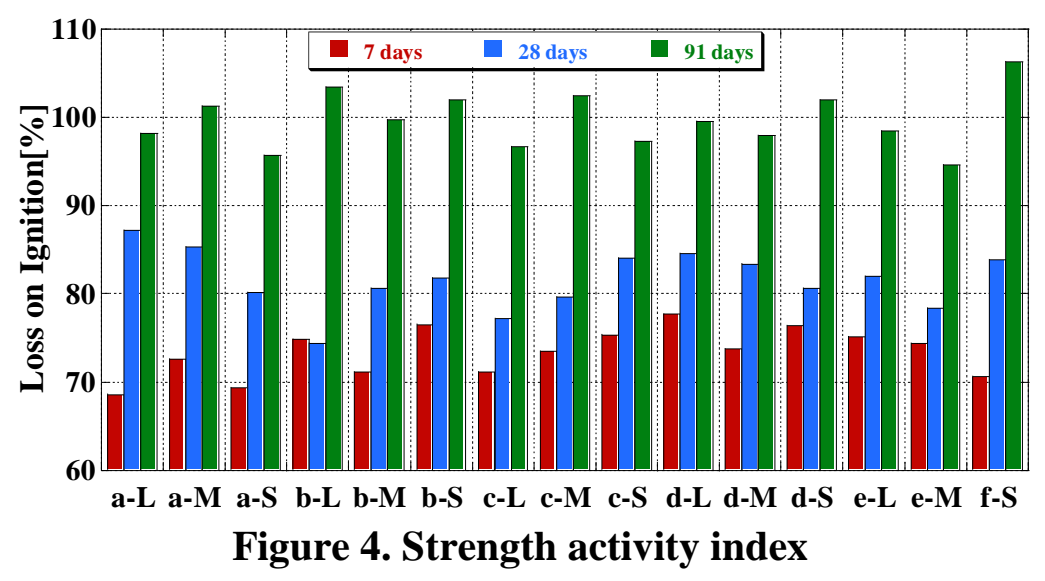

\section{CONCLUSION}

The following general conclusions can be drawn from the study provided in the paper:

- The possibility of meeting the JIS class II standard is high. It is clear that the flow value of CCAS can be appropriately evaluated by considering both ignition loss and BET specific surface area.

- We found that a BET specific surface area of greater than $3.60 \mathrm{~m} 2 / \mathrm{g}$ in the original fly ash (except fly ash type e) could be reduced to below $3.2 \mathrm{~m} 2 / \mathrm{g}$ by removing unburned carbon through modification of more than a medium modifying level.

- We found that immersing fly ash in water greatly affects the BET specific surface area and that the increase in the BET specific surface area by immersion points to the possibility of hydrate formation, which adversely affects the flow value.

\section{ACKNOWLEDGEMENT}

The experiment was supported by Eiji Mikura, Kazuyuki Miura, and Hayato Shoyama. We show gratitude to them.

\section{REFERENCE}

Koji Takasu, Experimental Study on Removal of Unburnt Carbon in Fly Ash by Flotation Method and Properties of Concrete with the Fly Ash Slurry, Architectual Institute of japan, pp331-340, $\operatorname{Japan}(2014)$

Syota Shimazaki, A Basic Study on Removing Unburnt Carbon in Fly Ash by Ore Flotation : Part 10 The influence by Long Storage of Reformed Fly Ash Slurry, Architectual Institute of japan, pp141144, Japan(2014) 